\title{
Development of Silicon Microelectrodes for Cochlear Implant Technology
}

Joanna R. Parker*, H. Barry Harrison**, Graeme M. Clark*, Jim Patrick*, Olaf Reinhold ${ }^{+}$

* Cooperative Research Centre for Cochlear Implant, Speech and Hearing Research, VIC.

** School of Microelectronic Engineering, Griffith University, QLD.

${ }^{+}$Defence Science and Technology Organisation, SA.

Abstract - Silicon fabrication technology is being explored as a possible solution to the manufacturing of advanced cochlear implant electrode arrays. Silicon probes have been produced with thickness of $5 \mu \mathrm{m}$ and coated in Parylene $\otimes$ polymer to provide strength. To enable handling they are given a backing of silicone rubber before surgical use. This paper presents some techniques used to produce such silicon microelectrodes.

\section{A. Introduction}

The inner ear, called the cochlea, is a spiral-shaped resonant cavity located in the temporal bone (Fig. 1) [1]. The temporal bone is the region of the skull at the side of the head, surrounding the ear. [2]

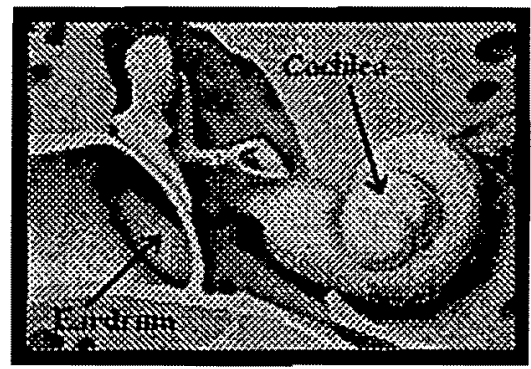

Figure 1: Middle and inner ear, showing cochlea.

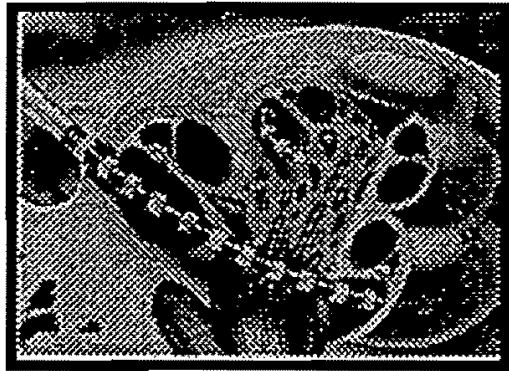

Figure 2: Placement of current technology electrode array inside cochlea. [3]

The structure and shape of the cochlea cause the frequencies of incoming sound waves to separate and spread along the length of the cochlea. Each frequency resonates at a different position within the cochlea and the resonating sound vibrations stimulate the nerves lying along the inner wall. The higher frequencies are detected closer to the entrance of the cochlea; the lower frequencies at the apex. The nerves form a pathway back to the hearing centre of the brain, allowing sounds to be perceived.

In some types of deafness, the mechanism responsible for converting frequencies into electrical nerve pulses does not function properly. The current treatment for this condition is to fit the patient with a cochlear implant.

A cochlear implant stimulates the nerves directly with electric charge generated by an external power source. An array of metal electrodes is inserted into the cochlea so that each is responsible for providing perception of a particular frequency (Fig 2). A processor is worn outside the body to select frequencies and intensities from the sounds ${ }^{-}$in the environment. These are converted into a code, and the information is relayed to the electrodes which stimulate the nerves accordingly, to provide the sensation of hearing. 
Currently cochlear implants are produced manually using a process developed prior to the availability of advanced microengineering technologies. 22 platinum rings forming the electrodes are manually welded to 22 platinum wires using a microscope and very fine instruments. This process is costly in terms of training and labour, and the yield depends very much on the dexterity of the person performing the assembly.

A new fabrication technique is proposed here which will exploit the benefits of silicon technology to produce not only cochlear implant electrodes, but of all nerve-stimulating microelectrodes in general. The technique will allow the production of microelectrodes with the following features:

-The ability to deliver or record the required stimulus with electrical characteristics equal to or better than any existing counterpart.

- To be constructed entirely of biocompatible materials, including the stimulation or recording sites, interconnections, mechanical carrier, and all-over protective coating.

-The materials used must remain biocompatible under operating conditions, and not deteriorate physically or electrically.

-To be strong enough so as to enable easy placement in the biological environment and shaped to minimise trauma both during placement and under operation.

- To be fabricated by a process which allows batch quantities and tailoring of the electrode shape to precise dimensions.

-To be fabricated by a process which is compatible with IC (integrated circuit) fabrication processes to allow easy integration with electronic circuitry for stimulating or recording.

\section{B. Experiment and Results}

To date two main techniques have been explored in an attempt to produce microelectrodes. Both require somewhat conventional lithographic techniques to delineate the microelectrode shape as shown in Figure 3.

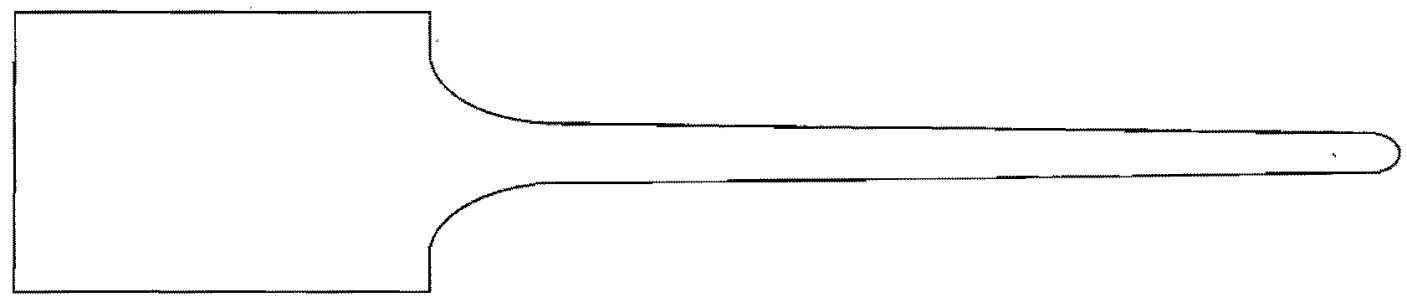

Figure 3: Shape of cochlear implant microelectrode.

The long thin section of the probe holds stimulating sites and is to be inserted into the cochlea. It is $400 \mu \mathrm{m}$ wide at the tip and $600 \mu \mathrm{m}$ wide where it joins to the large rectangular support. This large section supports bonding pads to enable the electrodes to connect to the stimulation circuitry. The whole probe is only about $5 \mu \mathrm{m}$ thick. At this thickness the silicon has the flexibility required to enable it to fit the shape of the cochlea.

To produce such an electrode, the first machining technique explored considered the etch shaping ability of boron doped silicon [4]. The depth profile for boron was calculated 
using the diffusion equation and associated diffusion coefficient calculations. Computer simulation also determined the thickness of masking oxide required to protect against diffusion of boron into unwanted areas.

Spin-on dopant was diffused for 2.5 hours, to give a doping depth of around $4 \mu \mathrm{m}$. The undoped silicon was then etched with a dopant-selective etchant, Potassium Hydroxide (KOH). After partial etching part of the sample was broken off and examined with a scanning electron microscope. The selectivity of the etchant between boron-doped silicon and nondoped silicon can be seen in Figure 4. The $\mathrm{KOH}$ etches the doped silicon at a much slower rate, so it can be seen as a 'cap' left on top of the remaining bulk silicon.

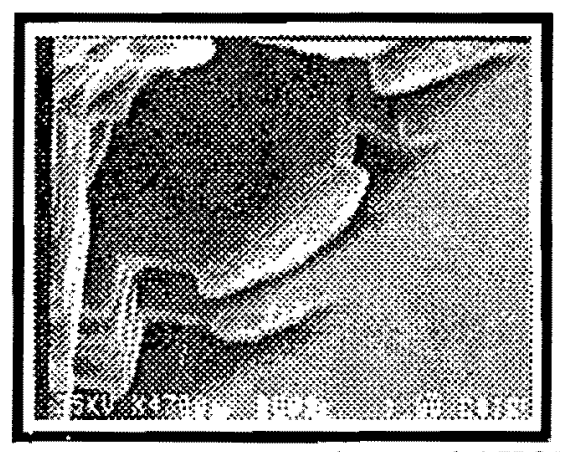

Figure 4: Boron-doped pattern after partial KOH etching.

At this stage the results appeared good, however after a long time in the etchant, a significant amount of the doped silicon was etched. The selectivity of the $\mathrm{KOH}$ did not allow it to etch through $300 \mu \mathrm{m}$ of non-doped silicon wafer and still leave the doped $4 \mu \mathrm{m}$ structures. However, after the whole etch, the structures remaining did resemble the original pattern.

The second approach has not been previously reported and involves the use of Silicon-on-Insulator (SOI) wafer. The wafer used was of the Buried Oxide (BOX), bonded type [5]. The microelectrodes were patterned into both the top silicon and buried oxide. The handle wafer was then etched from the back surface to release the probes. To date results indicate that this technique is more suitable for fabrication and handling than the boron etchstop approach.

Probes fabricated using the boron etch-stop technique were coated in Parylene 8 to provide strength and some stiffness. Some probes were given only $10 \mu \mathrm{m}$ per side, and others were given $20 \mu \mathrm{m}$ coating. They were then given a silicone rubber backing to aid in handling.

A cochlear implant surgeon tested the probes' mechanical properties by inserting them into pre-prepared cochleas. The probe coated in only $10 \mu \mathrm{m}$ of parylene was not stiff enough, making it difficult to insert and finally break. The $20 \mu \mathrm{m}$ coating performed much better, achieving a good insertion without breaking. The silicone backing was satisfactory for the surgeon to handle. However, its adhesion to the Parylene was not good, in some cases peeling away.

\section{Discussion}


Generally boron etch-stop is a flexible technique that allows precise machining in three dimensions. Its limitations are the long diffusion required and the difficulty of incorporating active circuitry on the machined device, due to the boron-doping.

In this experiment the boron etch-stop technique would have been more successful if the wafer had first been thinned from the back before using the $\mathrm{KOH}$ selective etch.

The SOI method which we believe to provide the most promise is currently being investigated using thicker buried oxide. It is believed that this technique is more compatible with the requirements outlined in the introduction.

The Parylene $\otimes$ coating was successful in that it enhanced the strength of the probes markedly. As there is $20 \mu \mathrm{m}$ of Parylene $B$ on each side of a $5 \mu \mathrm{m}$ probe, the Parylene $\otimes$ is predominately responsible for the mechanical properties of probe.

The use of silicone rubber to provide the backing is promising because silicone is biocompatible, convenient, readily available, and easy to use. It is anticipated that further research will provide a solution to aid in adhesion of silicone to Parylene 8 .

\section{Conclusion}

With the use of silicon fabrication processes, the future of microelectrodes will see a great deal of flexibility in their design and development. It will be possible to fabricate an almost unlimited range of microelectrodes, thereby opening up many more research paths in the areas of nerve stimulation and recording. The cochlear implant is just one example of an application which will greatly benefit from this technology.

\section{Acknowledgements}

Joanna Parker would like to acknowledge the support of the Cooperative Research Centre for Cochlear Implant, Speech and Hearing Research for their supervision and Griffith University also for supervision and for the use of facilities in this project.

\section{References}

[1] Arthur J. Vander, James H. Sherman, Dorothy S. Luciano, "Human Physiology: the mechanisms of body function" McGraw-Hill, 1994, USA.

[2] Henry Gray, "Gray's Anatomy", The Book Company, 1996, Sydney.

[3] Clark et al, "The University of Melbourne - Nucleus Multi-Electrode Cochlear Implant." Advances in Oto-Rhino-Laryngology, V. 38. C.R. Pfaltz (Ed.) Karger, Basel, 1987.

[4]David J. Anderson et al, "Batch-Fabricated Thin-Film Electrodes for Stimulation of the Central Auditory System", IEEE Trans. Biomed. Engr., pp. 693-704, July 1989.

[5]Sage Concepts (for SiBond), Title Unknown "Chap 3. Silicon on Insulator-(SOI)". 


\section{University Library}

\section{- M M I N E R VA A gateway to Melbourne's research publications}

Minerva Access is the Institutional Repository of The University of Melbourne

Author/s:

Parker, Joanna R.;Harrison, H. Barry;Clark, Graeme M.;Patrick, Jim;Reinhold, Olaf

Title:

Development of silicon microelectrodes for cochlear implant technology

Date:

1997

Citation:

Parker, J. R., Harrison, H. B., Clark, G. M., Patrick, J., \& Reinhold, O. (1997). Development of silicon microelectrodes for cochlear implant technology. In Proceedings of 1996 Conference on Optoelectronic and Microelectronic Materials and Devices, Canberra.

Persistent Link:

http://hdl.handle.net/11343/27005 